\title{
Double-slit Interference and Temporal Topos
}

\author{
Goro Kato and Tsunefumi Tanaka
}

The electron double-slit interference is re-examined from the point of view of temporal topos. Temporal topos (or t-topos) is an abstract algebraic (categorical) method using the theory of sheaves. A brief introduction to t-topos is given. When the structural foundation for describing particles is based on t-topos, the particle-wave duality of electron is a natural consequence. A presheaf associated with the electron represents both particle-like and wave-like properties depending upon whether an object in the site (t-site) is specified (particle-like) or not (wave-like). It is shown that the localization of the electron at one of the slits is equivalent to choosing a particular object in the $t$-site and that the electron behaves as a wave when it passes through a double-slit because there are more than one object in the t-site. Also, the single-slit diffraction is interpreted as a result of the possibility of many different ways of factoring a morphism between two objects.

\section{INTRODUCTION-THE ELECTRON DOUBLE-SLIT INTERFERENCE}

The electron double-slit interference is one of the most important experiments in physics. It shows both particle-like behavior and wave-like behavior of electrons. Also, the experiment shows statistical properties of quantum mechanics. The observed location of an individual electron on 


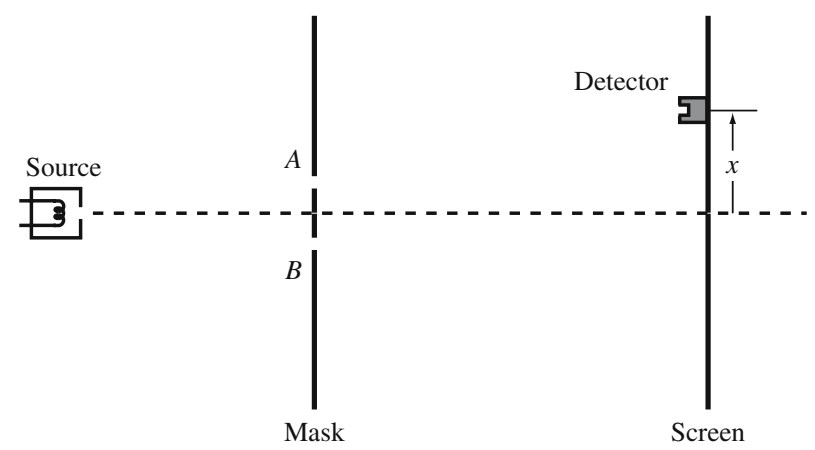

Fig. 1. Monoenergetic electrons fired from an electron gun pass through double-slits on the mask and arrive at a detector on the screen.

the screen is random, but an ensemble of identically prepared electrons exhibits a distribution resembling an optical interference pattern. This section of paper covers a brief review of the electron double-slit interference experiment including the description of apparatus, observations, and their implications on particle-wave duality.

\subsection{Experiment Setup}

In a typical setup, a beam of monoenergetic electrons from a source is incident on a mask with two narrow, parallel slits (labeled $A$ and $B$ in Fig. 1). The spacing between the slits is assumed to be much larger than the width of the slit. The rate at which electrons are emitted is so low that basically electrons arrive at the mask one at a time. This will eliminate a possibility that one electron passing through one slit may interfere with another electron through the second slit. After passing through the slits, electrons fall on a screen which is placed at a distance much greater than the spacing between the slits. On the screen is a particle detector which can be placed at various locations along the $x$-axis. The detector counts the number of electrons arriving at the position $x$.

\subsection{Observations}

For each electron shot toward the slits, always only one electron is registered by the detector somewhere on the screen. Positions of consecutive electrons on the screen show no apparent correlation between 


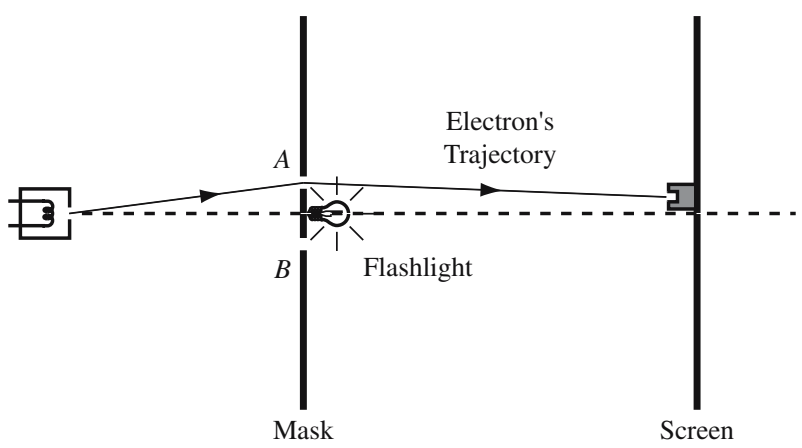

Fig. 2. A photon from a flashlight behind the mask is scattered by a passing electron revealing which slit the electron has passed through.

them, and the pattern at first appears random even though the electrons are emitted with an identical momentum from the source. As more and more electrons reach the screen, the probability density of the electrons' positions begins to resemble the interference pattern of monochromatic, coherent light passing through a double-slit. This outcome shows that an electron has some wave-like properties as it passes through the slits and that it behaves like a particle since it has a definite position when detected on the screen.

If an electron has a definite position at each instant of time on its way from the source to the screen, then it must go through one slit or the other. In order to find out which slit the electron passes through, a flashlight could be placed behind the mask (Fig. 2). A photon from the flash light is scattered by the electron after passing through one of the slits, and by measuring the direction into which the photon is deflected, the location where it interacted with the electron and thus which slit the electron passed through could be determined. Assuming every electron interacts with a photon, a probability distribution of electrons for each slit can be constructed. The result is the following: the probability density of finding the electrons that have passed through a certain slit is the same as the single-slit diffraction pattern of light on the screen. The distribution for each slit is identical to the one that would be expected if the other slit were completely covered. The total probability density in this experiment is a simple algebraic sum of the two noninterferring densities for individual slits. It is not the same as the probability density obtained in the original experiment in which the slit that electron passes through is unknown.

The interaction between electron and photon does change the electron's trajectory as the photon carries some momentum. Since the photon's 
momentum is inversely proportional to its wavelength, increasing its wavelength can decrease its disruptive effect on the electron's trajectory during the scattering. However, doing so increases the uncertainty in the measurement of the position where the electron-photon collision takes place. The interference pattern appears only for wavelengths greater than the order of the spacing between the slits, and with such a long-wavelength photon, the uncertainty becomes so large that which slit the electron has passed through can no longer be determined. ${ }^{(1)}$ To this date, every attempt to determine the electron's path and simultaneously maintain the interference pattern on the screen has failed. It appears that electron has both particlelike property and wave-like property, but we cannot observe both properties at the same time.

These apparently incompatible properties of electrons led Niels Bohr to propose the complementarity principle which states that it is impossible to describe the electron by the particle model alone or by the wave model alone. Both models are required to fully describe the electron. However, there exists a single, abstract algebraic object called temporal topos which can have both particle-like and wave-like properties. Temporal topos (or $t$-topos) could be a mathematical foundation for electrons exhibiting the particle-wave duality. In the next section, a concise introduction to $t$-topos is given.

\section{INTRODUCTION TO $T$-TOPOS AND PRESHEAFIFICATION OF OBSERVABLES}

We will give a brief introduction to the notions of a category and a (pre)sheaf. For a complete and precise description of the theories of categories and sheaves, we recommend Refs. 2 or 3.

\subsection{Category}

A category $\mathcal{C}$ consists of objects and morphisms. Let $\mathrm{Ob}(\mathcal{C})$ be the set (or class) of objects of $\mathcal{C}$. Then for $A, B \in \mathrm{Ob}(\mathcal{C}), \operatorname{Hom}_{\mathcal{C}}(A, B)$ is the set of all morphisms from $A$ to $B$. For $f \in \operatorname{Hom}_{\mathcal{C}}(A, B)$ and $g \in \operatorname{Hom}_{\mathcal{C}}(B, C)$ (which are often written as $f: A \longrightarrow B$ and $g: B \longrightarrow C$ ), the composition $g \circ f \in \operatorname{Hom}_{\mathcal{C}}(A, C)$ is defined. Then for $h: C \longrightarrow D$ we have $h \circ(g \circ$ $f)=(h \circ g) \circ f$. For each object $A$ of $\mathcal{C}$, there exists an identity morphism $1_{A}: A \longrightarrow A$ such that $1_{A} \circ f=f$ and $g \circ 1_{A}=g$ for any $f: B \longrightarrow A$ and $g: A \longrightarrow C$. 
Remark 1. In general, for a category $\mathcal{C}$, the dual category, denoted as $\mathcal{C}^{\text {opp }}$ or $\mathcal{C}^{\circ}$, has the same objects as the original category $\mathcal{C}$. However, a morphism $A \stackrel{f}{\longrightarrow} B$ in $\mathcal{C}$ becomes $A=A^{\circ} \stackrel{f^{\circ}}{\longleftarrow} B^{\circ}=B$ in $\mathcal{C}^{\text {opp }}$.

Example 1. Let $($ Sets) be the category of sets. Namely Ob(Sets) consists of sets, and morphisms in (Sets) are set-theoretic mappings.

Example 2. Let $(\mathcal{V e c})$ be the category of vector spaces over complex numbers $\mathbb{C}$. Then $\mathrm{Ob}(\mathcal{V e c})$ consists of vector spaces over $\mathbb{C}$, and morphisms of $(\mathcal{V e c})$ are $\mathbb{C}$-linear transformations.

Example 3. Let $(\mathcal{A} b)$ be the category of Abelian groups. Then $\mathrm{Ob}(\mathcal{A} b)$ consists of Abelian groups, and the morphisms in $(\mathcal{A} b)$ are Abelian group homomorphisms.

Next, let $\mathcal{C}$ and $\mathcal{C}^{\prime}$ be general categories. The product category $\mathcal{C} \times \mathcal{C}^{\prime}$ has its objects of the form $\left(A, A^{\prime}\right)$, where $A \in \mathrm{Ob}(\mathcal{C})$ and $A^{\prime} \in \mathrm{Ob}\left(\mathcal{C}^{\prime}\right)$. A morphism from $\left(A, A^{\prime}\right)$ to $\left(B, B^{\prime}\right)$ in $\mathcal{C} \times \mathcal{C}^{\prime}$ is a pair $\left(f, f^{\prime}\right)$ where $f: A \longrightarrow B$ in $\mathcal{C}$ and $f^{\prime}: A^{\prime} \longrightarrow B^{\prime}$ in $\mathcal{C}^{\prime}$.

We will define a functor from a category $\mathcal{C}$ to another category $\mathcal{D}$. A functor $F$ from $\mathcal{C}$ to $\mathcal{D}$, written as $F: \mathcal{C} \rightsquigarrow \mathcal{D}$, takes an object $A$ in $\mathcal{C}$ to an object $F A$ in $\mathcal{D}$, and also $F$ takes a morphism $f: A \longrightarrow B$ in $\mathcal{C}$ to a morphism $F f: F A \longrightarrow F B$ in $\mathcal{D}$. Then $F$ satisfies the following:

(1) For $f: A \longrightarrow B$ and $g: B \longrightarrow C$ in $\mathcal{C}, F(g \circ f)=F g \circ F f: F A \longrightarrow$ $F C$ in $\mathcal{D}$.

(2) For $1_{A}: A \longrightarrow A, F 1_{A}=1_{F A}: F A \longrightarrow F A$ in $\mathcal{D}$.

Remark 2. The functor $F$ is said to be a covariant functor if it does not change the direction of a morphism: $F A \stackrel{F f}{\longrightarrow} F B$. On the other hand, if the direction is reversed, $F A \stackrel{F f}{\longleftarrow} F B$, then $F$ is a contravariant functor.

\subsection{Presheaf}

The concept of a (pre)sheaf $F$ is classically defined as a contravariant functor $F$ from the associated category with a topological space $T$ to the category of sets. Namely, $F(U)$ is a set for any open subset $U$ of $T$. We need the following more flexible generalization of the above classical definition of a (pre)sheaf for our purpose. 
First, we will define the category induced by a topological space. Let $T$ be a topological space. For open sets $V$ and $U$ in $T$, if $V \subset U$ then define the set of morphisms from $V$ to $U$ to be $\{\iota\}$, where $\iota: V \hookrightarrow U$ is the inclusion mapping. If $V \not \subset U$, then define the set of morphisms from $V$ to $U$ to be an empty set. Next, define the category $\mathcal{T}$ associated with the topological space $T$ as follows: $\operatorname{Ob}(\mathcal{T})$ consists of all open sets in $T$, and for $V, U \in \operatorname{Ob}(\mathcal{T}), \operatorname{Hom}_{\mathcal{T}}(V, U)=\{\iota\}$ where $V \stackrel{\iota}{\hookrightarrow} U$ for $V \subset U$, and $\operatorname{Hom}_{\mathcal{T}}(V, U)=\varnothing$ for $V \not \subset U$. Then the definition of a presheaf is the following. A presheaf $F$ is a contravariant functor from $\mathcal{T}$ to a category $\mathcal{C}$. The category of presheaves on $\mathcal{T}$ is simply $\mathcal{C}^{\mathcal{T}^{\circ}}$ where $\mathcal{T}^{\circ}$ is the dual category of $\mathcal{T}$.

Remark 3. Let $\mathcal{C}$ and $\mathcal{C}^{\prime}$ be categories. A contravariant functor $F: \mathcal{C} \rightsquigarrow$ $\mathcal{C}^{\prime}$ is a covariant functor $F: \mathcal{C}^{\circ} \rightsquigarrow \mathcal{C}^{\prime}$. A contravariant functor $G: \mathcal{C} \rightsquigarrow \mathcal{C}^{\prime}$ is a covariant functor $G: \mathcal{C} \rightsquigarrow \mathcal{C}^{\prime \circ}$.

For our $t$-topos, we replace $\mathcal{T}$ by a general site $\mathcal{S}$ and also replace $\mathcal{C}$ by a product category of categories $\mathcal{C}_{\alpha}, \alpha \in \Gamma$, where $\Gamma$ is an index set. Namely, we consider $\left(\prod_{\alpha \in \Gamma} \mathcal{C}_{\alpha}\right)^{\mathcal{S}^{\circ}}$.

\subsection{Site}

Since we need more than one morphism between two objects in the initial category, we need to consider a site. A site is a category with a Grothendieck topology, and its definition is as follows. Let $\mathcal{S}$ be a category. Then $\mathcal{S}$ is said to be a site if there exists a set $\operatorname{Cov}(\mathcal{S})$ of coverings (families of morphisms) $\left\{U_{i} \stackrel{l_{i}}{\longrightarrow} U\right\}$ in $\mathcal{S}$ satisfying the following conditions:

(1) An isomorphism $U^{\prime} \stackrel{\iota^{\prime}}{\longrightarrow} U$ is a covering of $U$ and thus an element of $\operatorname{Cov}(\mathcal{S})$.

(2) Let $V \stackrel{f}{\longrightarrow} U$ be a morphism in $\mathcal{S}$. For a covering $\left\{U_{i} \stackrel{\iota_{i}}{\longrightarrow} U\right\}_{i \in I}$, $\left\{U_{i} \times_{U} V \longrightarrow V\right\}$ is a covering of $V$. The commutative diagram for this is shown in Fig. 3.

(3) For a covering $\left\{U_{i} \stackrel{\iota_{i}}{\longrightarrow} U\right\}$ in $\mathcal{S} \in \operatorname{Cov}(\mathcal{S})$, if $\left\{V_{i j} \stackrel{\iota_{i j}}{\longrightarrow} U_{i}\right\}$ is a covering of $U_{i}$, then the family of morphisms obtained by the composition $\left\{V_{i j} \stackrel{{ }_{i} \circ l_{i j}}{\longrightarrow} U\right\}_{j \in J_{i}, i \in I}$ is a covering of $U$.

A set of morphisms $\left\{U_{i} \longrightarrow U\right\}$ which satisfies (1)-(3) is called a covering of $U$. Thus, $\operatorname{Cov}(\mathcal{S})$ is a set of coverings of the object $U$ for $U \in \mathcal{S}$, and it should be written as 


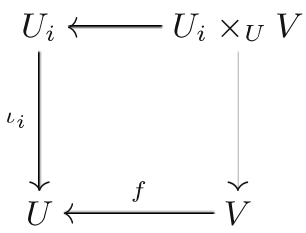

Fig. 3. Covering of $V$.

$$
\operatorname{Cov}(\mathcal{S})=\left\{\left\{U_{i} \longrightarrow U\right\}_{i \in I ; U \in \mathcal{S}}\right\}
$$

The site $\mathcal{S}$ is a category with the set $\operatorname{Cov}(\mathcal{S})$, and it is often called a category with a Grothendieck topology (for more on site, see Refs. 2-5).

\section{4. $t$-Topos and Presheafification of Physical Quantities}

A temporal topos, or $t$-topos, is the category $\hat{\mathcal{S}}$ of presheaves over a site $\mathcal{S}$ (which we call the $t$-site) to a product category of categories indexed by a set $\Gamma{ }^{1}$ Namely,

$$
\hat{\mathcal{S}}=\left(\prod_{\alpha \in \Gamma} \mathcal{C}_{\alpha}\right)^{\mathcal{S}^{\mathrm{opp}}}
$$

The objects $\operatorname{Ob}(\hat{\mathcal{S}})$ of category $t$-topos consist of $\left(m_{\alpha}\right)$, indexed by the index set $\Gamma$, where each $m_{\alpha}$ is a presheaf over the $t$-site $\mathcal{S}$ (i.e., $m$ is a contravariant functor from $\mathcal{S}$ to the product category $\prod_{\alpha \in \Gamma} \mathcal{C}_{\alpha}$ ). Morphisms in the $t$-topos consist of natural transformations $\left(s_{\alpha}\right)$, indexed by $\Gamma$, between presheaves $\left(m_{\alpha}\right)$ and $\left(m_{\alpha}^{\prime}\right)$; i.e., in each $\mathcal{C}_{\alpha}, \alpha \in \Gamma$,

$$
s_{\alpha}: m_{\alpha} \longrightarrow m_{\alpha}^{\prime}
$$

is a morphism between contravariant functors $m_{\alpha}$ and $m_{\alpha}^{\prime}$. More explicitly,

$$
s_{\alpha}(V): m_{\alpha}(V) \longrightarrow m_{\alpha}^{\prime}(V)
$$

is a morphism from the object $m_{\alpha}(V)$ to the object $m_{\alpha}^{\prime}(V)$ for an object $V$ in $t$-site $\mathcal{S}$.

\footnotetext{
${ }^{1}$ The name " $t$-topos" for the category of presheaves from a $t$-site has been used simply as a terminology at this elemental stage of our theory. However, when the notion of $t$-topos is more fully developed, the name $t$-topos may be evident and more clarified.
} 
The target category is the product category $\prod_{\alpha \in \Gamma} \mathcal{C}_{\alpha}$, indexed by $\Gamma$, whose components are discrete categories $\mathcal{C}_{2}, \mathcal{C}_{1}, \mathcal{C}_{\text {Planck }}, \ldots$, where 2, 1 , Planck $\in \Gamma$ (i.e., categories with no morphisms except identity morphisms) and nondiscrete categories where measurements (morphisms) may exist over certain $t$-site objects. Note also that in general the dual (opposite) category is often used when contravariant nature is changed to covariant nature. As for $t$-topos, the (fixed) $t$-site $\mathcal{S}$ is replaced by $\mathcal{S}^{\text {opp }}$ so that objects in $t$-topos $\hat{\mathcal{S}}$ become covariant functors from the dual $\mathcal{S}^{\text {opp }}$ to the target category $\prod_{\alpha \in \Gamma} \mathcal{C}_{\alpha}$ (for a more precise description of $t$-topos theory, see Refs. 5 and 6 ).

A physical system under study is to be represented by $\hat{\mathcal{S}}$. Let $\mathcal{C}_{1}$ be the discrete category of particles. An object of $\mathcal{C}_{1}$ is a particle in microcosm, and as a category, $\mathcal{C}_{1}$ is discrete; i.e., the only morphisms in $\mathcal{C}_{1}$ are the identities $1_{X}$ such that $X \stackrel{1_{X}}{\longrightarrow} X$ for $X \in \mathrm{Ob}\left(\mathcal{C}_{1}\right)$. Here the microcosm includes physical properties, such as positions and momenta of individual particles at the quantum level. In contract, macroscopic properties of the system at the classical level form the macrocosm category $\mathcal{C}_{2}$.

It is possible to introduce another category $\mathcal{C}_{\text {Planck }}$ below the quantum level $\left(\mathcal{C}_{\text {quantum }}\right)$ to describe phenomena at the Planck scale and even those at the sub-Planck scale (see Ref. 7 for the $t$-topos theoretic definition of a sub-Planck object). However, a (ur-)particle, as the direct limit object, at the (sub)Planck level is only a categorically defined universal object (i.e., the object satisfying the universal mapping property in the category) (see Refs. 3, 7, and 8 for direct and inverse limits). It is our intention to describe observable physical entities such as particles, position, time, and energy with presheaves. We do not need delicate topological properties of the $t$-site for microcosmic study. However, we expect that topology of the $t$-site will play an important role for the study of singularities and related topics in general relativity.

In order to describe an electron $\underline{e}$, we associate with it a presheaf $e$ in the following sense: there exists an object $V$ in the $t$-site $\mathcal{S}$ such that in $\mathcal{C}_{1}$,

$$
\underline{e}=e(V) \text {. }
$$

Then we define that the presheaf $e$ is in a "particle ur-state" when an object $V$ is chosen as in Eq. (5) and thus in a localized state. It can be said that the electron presheaf $e$ is manifested into a particle $\underline{e}$ when the object $V$ is specified. The electron is not in a particle state in the usual sense. An observation of the electron resulting in real numbers associated with the electron's position, energy, and momentum involves a transfer of information from $e(V)$ to a presheafified observer, and it is explained in the next section. 
When an object of $\mathcal{S}$ is not specified for $e$ (i.e., $e$ is in a nonlocalized state), $e$ is said to be in a "wave ur-state," written as either $\{e(V)\}_{V \in \operatorname{Ob}(\mathcal{S})}$ or $e(-)$. Note that $e(-)$ may be interpreted as a wave function-like entity which collapses into a localized particle when the object $V$ is specified. Furthermore, it is possible to consider $e$ as being composed of several presheaves which are to be evaluated at objects of $\mathcal{S}$. This notion of the wave ur-state will be expanded later when the double-slit interference experiment is examined. And for the fundamental concepts in $t$-topos (see Refs. 5 and 6). Also, the application of $t$-topos to nonlocal quantum entanglement in the EPR-type experiments can be found in Refs. 8 and 9.

\subsection{Presheafification of Time and Space}

For an electron $\underline{e}$ in $\mathcal{C}_{1}$, time $t$ and position $x$ are associated with it locally. Let $\tau$ and $\kappa$ be presheaves associated with time and space, respectively. That is, we presheafify time and space as in Refs. 5 and 6 . The presheaves $\tau$ and $\kappa$ are objects of $\hat{\mathcal{S}}$ and are defined over a common object in the $t$-site $\mathcal{S}$ so that physical time and space, $t$ and $x$, are related to $\tau$ and $\kappa$ by

$$
t=\tau(V) \quad \text { and } \quad x=\kappa(V) .
$$

The object $V$ of the $t$-site $\mathcal{S}$ can be considered as a generalized time period. What one observes in $\mathcal{C}_{1}$ is a "slice" (or the microcosm component) of the associated presheaf evaluated at a certain generalized time period of $\mathcal{S}$. A pair $(\tau(V), \kappa(V))$ plays a role of a local coordinate system of $e(V)$ (see Ref. 7 for the relativistic version of $t$-topos).

The coordinate pair $(\tau, \kappa)$ can be considered as one object of $\hat{\mathcal{S}}$, and since it is associated with a certain electron presheaf $e$, it should be denoted as $\left(\tau_{e}, \kappa_{e}\right)$ to clarify its affiliation with $e$ (see also Refs. 6 and 7 for the dependency of presheaves $\tau$ and $\kappa$ on a particle (the associated presheaf)). In general, if $m_{1}, m_{2}, \ldots, m_{r}$ are objects of $\hat{\mathcal{S}}$ and if the $r$-tuple $\left(m_{1}, m_{2}, \ldots, m_{r}\right)$ can be considered as one object of $\hat{\mathcal{S}}$ over a restricted subset of the set of objects in $\mathcal{S}$, then $m_{1}, m_{2}, \ldots, m_{r}$ are said to be "ur-entangled." That is, $m_{i}$, where $i=1,2, \ldots, r$, are defined on the same object of the $t$-site $\mathcal{S}$. For example, if two electrons $e$ and $e^{\prime}$ are correlated, then the system at a common generalized time $V$ can be described by an object $\left(e(V), e^{\prime}(V)\right)$ (see Ref. 9 for the application of $t$-topos to the EPRtype nonlocality).

As already introduced earlier, we propose the generalized time $V$, which is an object in site $\mathcal{S}$, as a more primitive notion of time than the 


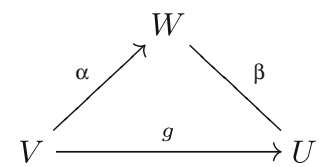

Fig. 4. Factorization of the morphism $g$.

physical time $t$. If we defined the physical time as $t=\tau(V)$, where $\tau$ is the time presheaf, then time is of local nature in the sense that for any object $V$ in $\mathcal{S}, \tau(V)$ may exist only locally and may not be globally extended, just like a particle being a locally defined entity as compared to a wave which is an extended (nonlocal) entity.

For the time presheaf $\tau$, suppose $\tau(V)$ precedes $\tau(U)$ and there exits a morphism $g: V \longrightarrow U$ in $\mathcal{S}$. "An electron $\underline{e}$ is emitted from the source, and then the electron hits the screen" can be phrased as follows in terms of $t$-topos: let $e$ be the presheaf associated with the electron and let $V$ be an object of $\mathcal{S}$ determining the particle ur-state of $e$ when emitted. Let also $U$ be an object of $\mathcal{S}$ determining the particle ur-state of $e$ when the electron hits the screen. ${ }^{2}$ Namely, $e(V)$ and $e(U)$ are observed and $\tau(V)$ precedes $\tau(U)$. In the $t$-site $\mathcal{S}$ we have

$$
g: V \longrightarrow U
$$

Next define $\{V \stackrel{g}{\longrightarrow} U\}$ be the set of all objects $W$ and morphisms in $\mathcal{S}$ that factor $g: V \longrightarrow U$ in $\mathcal{S}$. That is, an element $(\alpha, W, \beta)$ of the set $\{V \stackrel{g}{\longrightarrow} U\}$ is given by the commutative diagram in $\mathcal{S}$ satisfying $g=\beta \circ \alpha$ (see Fig. 4). Consequently, once an electron is emitted, the electron is in a wave ur-state $\{e(W)\}_{W \in \mathrm{Ob}(\mathcal{S})}$ until it hits the screen, unless the electron is observed during a time interval between $\tau(V)$ and $\tau(U)$.

\subsection{Observations and Transfer of Information}

The definition of observation of a presheaf $e$ by an observer or an instrument represented by another presheaf $P$ is the following. There exists a nondiscrete category $\mathcal{C}_{\alpha}, \alpha \in \Gamma$ such that in $\mathcal{C}_{\alpha}$ there is a morphism

\footnotetext{
${ }^{2}$ For ur-states $e(V)$ at an initial time $\tau(V)$ and $e(U)$ at a later time $\tau(U)$, there correspond quantum states $\left|\psi_{V}\right\rangle$ and $\left|\psi_{U}\right\rangle$, respectively. If $g$ is the morphisms from $V$ to $U$ in the $t$-site $\mathcal{S}$, the presheaf $e$ induces the evolution morphism $e(g)$ from $e(U)$ to $e(V)$. Corresponding to $e(g)$ is a unitary operator $\mathcal{U}_{V \rightarrow U}$ such that $\left|\psi_{U}\right\rangle=\mathcal{U}_{V \rightarrow U}\left|\psi_{V}\right\rangle$. The evolution operator $\mathcal{U}_{V \rightarrow U}$ is similar to the time evolution operator in the usual quantum mechanical sense.
} 


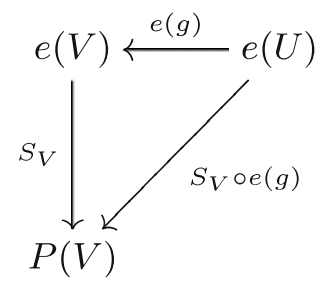

Fig. 5. Observation of $e$ by $P$ over $V$.

$$
S_{V}: e(V) \longrightarrow P(V)
$$

for some object $V$ in site $\mathcal{S}$ when the electron $e$ and the observer $P$ interact. The morphism $S_{V}$ is a natural transformation over the generalized time period $V$, and the natural transformation is a morphism of functors (between $e$ and $P$ in our case). This definition of an observation implies that a wave ur-state $\{e(V)\}_{V \in \mathrm{Ob}(\mathcal{S})}$, or $e(-)$, must collapse to a particle ur-state by specifying an object $V$ of $\mathcal{S}$ in order to be measured by the observer $P$.

Under the same notations as in Sec. 2.5, now the question is how much information $P$ can get about the ur-state $e(U)$ by measuring $e(V)$. In order to answer this question, we first consider the commutative diagram shown in Fig. 5. The morphism $e(g): e(U) \longrightarrow e(V)$ is induced from Eq. (7) by the presheaf $e$. (Since a presheaf is a contravariant functor by definition, the direction of the arrow, $V \stackrel{g}{\longrightarrow} U$, is reversed, $e(V) \stackrel{e(g)}{\longleftarrow} e(U)$, when the morphism $g$ in $\mathcal{S}$ is carried over to the product category $\prod_{\alpha \in \Gamma} \mathcal{C}_{\alpha}$ by the presheaf $e$.)

We consider the image, $\operatorname{Im}\left(S_{V}\right)$, of the morphism $S_{V}$ to be information $P$ received over $V$ by observing $e(V)$. The image $\operatorname{Im}\left(S_{V}\right)$ is a subobject of $P(V)$ which is influenced by $e(V)$. Then we have that

$$
\operatorname{Im}\left(S_{V}\right) \supset \operatorname{Im}\left(S_{V} \circ e(g)\right) .
$$

The composite morphism $S_{V} \circ e(g)$ from $e(U)$ to $P(V)$ is an indirect observation of $e(U)$ by $P(V)$. The information from $e(U)$ as represented by the image $\operatorname{Im}\left(S_{V} \circ e(g)\right)$ is never greater than $\operatorname{Im}\left(S_{V}\right)$. The inclusion " $\supset$ " in Eq. (9) should be interpreted as:

(1) In general, information about the past (ur-)state $e(V)$ does not provide the entire information of the future (ur-)state $e(U)$. One can obtain only a partial knowledge of $e(U)$ by measuring $e(V)$. 


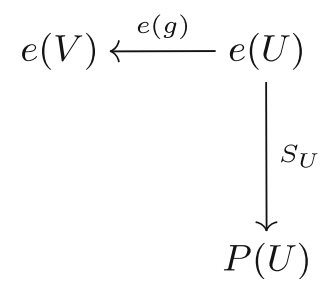

Fig. 6. Observation of $e$ by $P$ over $U$.

(2) Consider the commutative diagram in Fig. 6. Namely, when the future (ur-)state $e(U)$ is measured, one does not get any information about the past (ur-)state $e(V)$.

(See Note 1.7 in Ref. 6 relating Isham-Butterfield's work ${ }^{(10-13)}$ for how values may be assigned to physical quantities in quantum mechanics from the presheaves via Kochen-Specker theorem, especially 2.2 in Ref. 11 at the level of logic.)

\section{THE DOUBLE-SLIT INTERFERENCE IN TERMS OF T-TOPOS}

\subsection{Factorization}

Suppose an electron is observed at the initial position $\left(t_{i}, x_{i}\right)$ then later at $\left(t_{f}, x_{f}\right)$. No observation is made between $x_{i}$ and $x_{f}$. The actual trajectory of the electron is unknown because its position was not measured between the two positions.

Let $V$ be an object associated with the first observation of the electron at $\left(t_{i}, x_{i}\right)$ and $U$ with the second observation at $\left(t_{f}, x_{f}\right)$. Then,

$$
t_{i}=\tau(V), \quad x_{i}=\kappa(V), \quad \text { and } \quad t_{f}=\tau(U), \quad x_{f}=\kappa(U),
$$

where $\tau$ and $\kappa$ are time and position presheaves. When the electron is localized by observation, that is when an object is specified, the time presheaf $\tau$ becomes the physically observable time $t$. The first observation precedes the second one, which is written as $\tau(V)<\tau(U)$.

Suppose the electron's position is measured once somewhere on its way to the final position, and it is at $\left(t_{1}, x_{1}\right)$. Let $W_{1}$ be an object associated with the observation at $\left(t_{1}, x_{1}\right)$ so that 


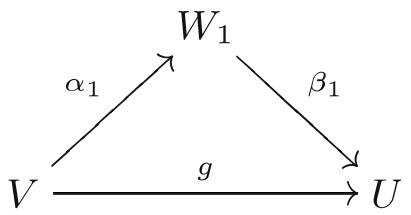

Fig. 7. An intermediate observation between the final and initial positions causes a factorization of $g$ and a localization of the electron.

$$
t_{1}=\tau\left(W_{1}\right), \quad x_{1}=\kappa\left(W_{1}\right)
$$

and the order of observations satisfies $\tau(V)<\tau\left(W_{1}\right)<\tau(U)$. A new commutative diagram corresponding to this series of observations is shown in Fig. 7 where $\alpha_{1}$ is the morphism from $V$ to $W_{1}$ and $\beta_{1}$ is the morphism from $W_{1}$ to $U$. In this case, $g$ has been factored into $g=\beta_{1} \circ \alpha_{1}$. If the electron is observed at a different location, say $\left(t_{2}, x_{2}\right)$, then there exist an object $W_{2}$, which is different from $W_{1}$, such that $t_{2}=\tau\left(W_{2}\right)$ and $x_{2}=\kappa\left(W_{2}\right)$. In general, for each intermediate observation of the electron between the initial position and the final position, there exists an object $W_{j}$ in $\mathcal{S}$ and a factorization of $g$ (see Fig. 8):

$$
g=\beta_{j} \circ \alpha_{j}
$$

The only restriction imposed on the intermediate position $\left(t_{j}, x_{j}\right)=\left(\tau\left(W_{j}\right)\right.$, $\left.\kappa\left(W_{j}\right)\right)$ is that the electron can physically be at that position and that the observation takes place after the initial observation but before the final observation, $\tau(V)<\tau\left(W_{j}\right)<\tau(U)$. $^{3}$

Furthermore, each intermediate morphism, $\alpha_{j}$ or $\beta_{j}$, can be factored as well. For example, if an observation is made between $V$ and $W_{j}$ and if

\footnotetext{
${ }^{3}$ For each factorization of $g$ with an intermediate object $W_{j}$ in $t$-site $\mathcal{S}$, the electron presheaf $e$ induces $e(g)=e\left(\beta_{j}\right) \circ e\left(\alpha_{j}\right)$ where $e\left(\alpha_{j}\right)$ is an evolution morphism from $e\left(W_{j}\right)$ to $e(V)$ and $e\left(\beta_{j}\right)$ is an evolution morphism from $e(U)$ to $e\left(W_{j}\right)$. Corresponding to $e\left(\alpha_{j}\right)$ and $e\left(\beta_{j}\right)$ are the evolution operators $\mathcal{U}_{V \rightarrow W_{j}}$ and $\mathcal{U}_{W_{j} \rightarrow U}$, respectively. The quantum state evolves from $\left|\psi_{V}\right\rangle$ to $\left|\psi_{W_{j}}\right\rangle$ then to $\left|\psi_{U}\right\rangle$ by a sequence of the evolution operators: $\mathcal{U}_{V \rightarrow U}=\mathcal{U}_{W_{j} \rightarrow U} \mathcal{U}_{V \rightarrow W_{j}}$. If the electron is not observed between the source and the screen, that is, if no intermediate object $W_{j}$ between $V$ and $U$ in $\mathcal{S}$ is chosen, then the morphism $g$ may have various factorizations. Therefore, the corresponding evolution operator $\mathcal{U}_{V \rightarrow U}$ includes all allowed sequences of the evolution operators from the state $\left|\psi_{V}\right\rangle$ to $\left|\psi_{U}\right\rangle$. Each sequence corresponds to a particular evolutionary path taken by the electron from the source to the screen.
} 


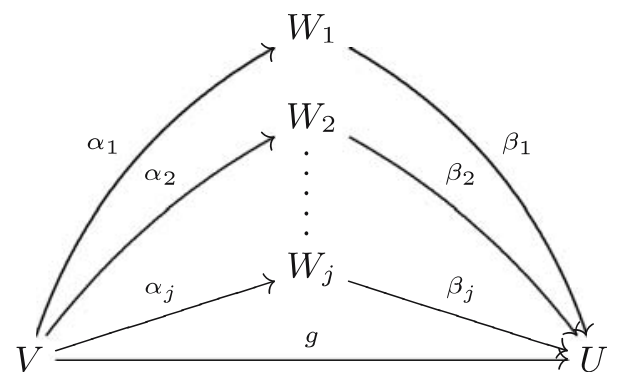

Fig. 8. The morphism $g$ from $V$ to $U$ can be factored into many ways as long as the intermediate position of the electron $\left(\tau\left(W_{j}\right), \kappa\left(W_{j}\right)\right)$ is within the light cone of the preceding observed position.

$Y_{j k}$ is an object associated with this observation, then $\alpha_{j}$ can be factored into $\alpha_{j}=\delta_{j k} \circ \gamma_{j k}$ (see Fig. 9).

Next, suppose a mask with two parallel slits is placed between the initial position and the final position of the electron. The electron is allowed to travel from one side of the mask to the other side only through the slits $A$ and $B$ as shown in Fig. 1. The presence of the double-slit reduces the unrestricted commutative diagram in Fig. 8 to the fundamental commutative diagram for the double-slit interference shown in Fig. 10. The objects $W_{A}$ and $W_{B}$ are associated with possible observations of the electron at the slits $A$ and $B$, respectively. Each morphism $\alpha_{A}, \alpha_{B}, \beta_{A}$, and $\beta_{B}$ can be further factored because there is no restriction in the position of the electron anywhere between the source and the slit and between the slit and the screen.

Although it is known with certainty that the electron does pass through the slits to reach the screen, which slit the electron actually passes through is unknown because no observation is made at the slit to detect its presence. Figure 10 shows that $g$ can be factored into $\beta_{A} \circ \alpha_{A}$ and $\beta_{B} \circ \alpha_{B}$. However, because no observation is made at the double-slit and because there is more than one object that corresponds to a possible position of the electron at the double-slit, the electron is in a nonlocalized state there and behaves as a wave ur-state according to $t$-topos (see Refs. 5 and 6). The object $U$ for the electron presheaf on the screen is influenced by $W_{A}$ and $W_{B}$ at the slits. It is analogous to the superposition principle for waves. In $t$-topos, when the electron is detected at the screen, it is in the particle ur-state $e(U)$. But because the object is not specified at the double-slit, $U$ and thus $e(U)$ are dependent on both $W_{A}$ and $W_{B}$. 


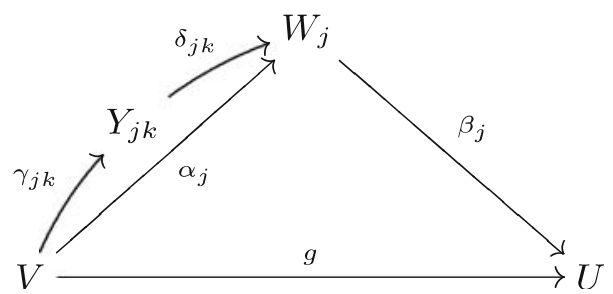

Fig. 9. Each morphism can be factored many times as more observations are made.

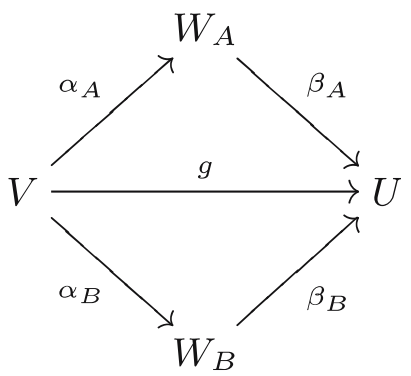

Fig. 10. The most fundamental commutative diagram for an electron undergoing the doubleslit interference. The electron is not detected at either slit.

If the electron is detected at the slit $A$ with a setup like the one shown in Fig. 2, then the factorization of $g$ must be from $V$ to $U$ via $W_{A}$ (i.e., $g=\beta_{A} \circ \alpha_{A}$ ) but not via $W_{B}$, and the fundamental commutative diagram (Fig. 10) reduces to a simpler diagram with only one possible factorization of $g$ as shown in Fig. 11, namely, the only upper triangle commutativity. This commutative diagram can also describe an experiment in which the slit $B$ is completely closed. In such a case, all possible trajectories from the source to a point on the screen must go through the slit $A$. Then, the morphism $g$ factors into $V \stackrel{\alpha_{A}}{\longrightarrow} W_{A} \stackrel{\beta_{A}}{\longrightarrow} U$ even though no actual observation of the electron is made at the slit $A$.

On the other hand, if the fired electron is not detected at the slit $A$, then it must have gone through the slit $B$ assuming that the detector never misses the electron through the slit. Therefore, null detection of the electron at the slit $A$ implies that $g$ factors into $\beta_{B} \circ \alpha_{B}$, and the fundamental commutative diagram reduces to the one shown in Fig. 12. 


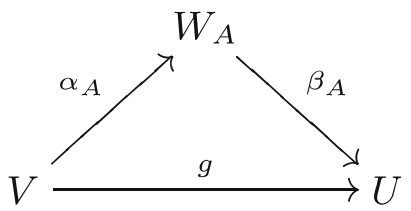

Fig. 11. The commutative diagram corresponding to the experiment in which the electron is observed at the source, then at the slit $A$, and finally on the screen.

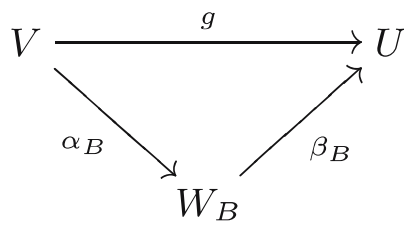

Fig. 12. The commutative diagram corresponding to the experiment in which it is known with certainty that the electron passes through the slit $B$ or that it does not pass through the slit $A$.

It is possible that the detector at the slit $A$ is not $100 \%$ reliable so that the electron could have evaded detection at the slit. In such a case, the commutative diagram for the experiment could be either Fig. 12 or Fig. 10. Also, if the wavelength of photon used to detect the position of the electron at the double-slit is in the same order as the slit spacing, then which slit the electron passes through is undetermined. Although an observation is made in these cases, the associated object at the slit cannot be assigned. The fundamental diagram for the double-slit (Fig. 10) cannot be reduced to either single-slit diagrams (Fig. 11 or Fig. 12). Hence, the electron remains in a wave ur-state at the double-slit.

\subsection{Single-slit Diffraction}

Even when one of the slits is closed or when the electron is detected at one of them, say slit $A$, the electron can still behave as a wave and produce a diffraction pattern on the screen. The slit $A$ has a finite width so it can be considered as consisting of several smaller subslits, indexed by $\ell$. Temporal-topos implies that for each subslit there exists an associated 


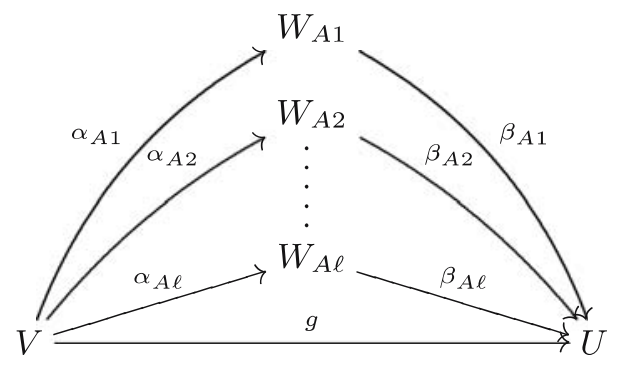

Fig. 13. The commutative diagram corresponding to the single-slit diffraction of an electron by a finite-width slit. The slit has been divided into several subslits, each with an associated object $W_{A \ell}$ and a factorization $g=\beta_{A \ell} \circ \alpha_{A \ell}$.

object $W_{A \ell}$. In other words, the commutative diagram (Fig. 11) can be drawn as a more detailed diagram (Fig. 13) showing the factorization $g$ within the slit $A$. There is more than one way to factor $g$ at the slit so that the electron is unlocalized within the width of the list. The object $U$ is influenced by all intermediate objects $\left\{W_{A \ell}\right\}$ associated with the slit $A$. That means, the electron acts like a wave, and therefore it is diffracted by the single-slit.

\subsection{Statistical Ensemble}

What has been described above applies to one electron only. When an electron is fired from the source, there is an associated object $V$. The observation of this electron on the screen is associated to another object $U$. There is one morphism $g$ from $V$ to $U$. Here, $g, V$, and $U$ are all associated with this electron. If another electron were fired, it would be associated with different objects, morphism, and presheaves. Several electrons may end up at the same final position on the screen $x_{f}$ after they are fired one by one, but their associated objects are different. For example, let $x_{f}^{(1)}$ be the final position of the first electron and $x_{f}^{(2)}$ be the final position of the second electron. Then,

$$
x_{f}^{(1)}=\kappa^{(1)}\left(U^{(1)}\right), \quad x_{f}^{(2)}=\kappa^{(2)}\left(U^{(2)}\right) .
$$

Notice that the position presheaf $\kappa^{(1)}$ represents only the first electron and $\kappa^{(2)}$ represents only the second electron. Suppose both electrons are detected at the same position on the screen, i.e., $x_{f}^{(1)}=x_{f}^{(2)}$. This does not 


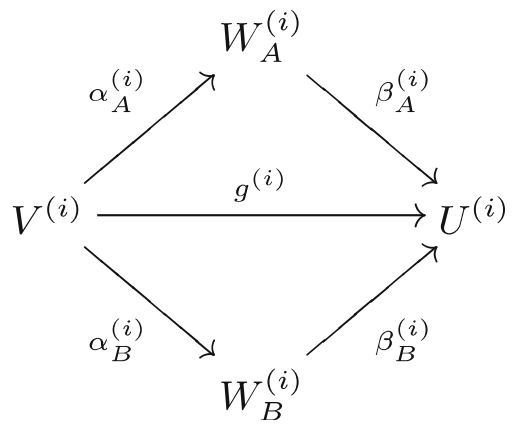

Fig. 14. The most fundamental commutative diagram for the $i$ th electron in an ensemble of identically prepared electrons undergoing the double-slit interference.

imply the objects $U^{(1)}$ and $U^{(2)}$ are identical in $t$-site. This is because $U^{(1)}$ is associated with the observation of the first electron and $U^{(2)}$ with the observation of the second electron.

Next, consider similarly prepared, identical electrons being fired repeatedly from the source. The $i$ th electron in this ensemble is associated with objects $V^{(i)}, U^{(i)}$, and morphism $g^{(i)}$. The commutative diagram for the $i$ th electron is shown in Fig. 14. This diagram belongs to only the $i$ th electron. For the ensemble of $N$ identical electrons, a set of final vertical positions on the screen can be formed, $\left\{x_{f}^{(1)}, x_{f}^{(2)}, \ldots, x_{f}^{(N)}\right\}$. If the electrons are not detected at the double-slit, the statistical distribution of the final positions should exhibit the double-slit interference pattern. This is because each electron is in the wave ur-state between $V^{(i)}$ and $U^{(i)}$ and because $U^{(i)}$ is influenced by $W_{A}^{(i)}$ and $W_{B}^{(i)}$ at the screen.

On the other hand, if the electrons are detected at the double-slit, and which slit (either $A$ or $B$ ) each electron passes through is known with certainty, then the set of the final positions $\left\{x_{f}^{(1)}, x_{f}^{(2)}, \ldots, x_{f}^{(N)}\right\}$ becomes a disjoint union of two sets, one associated with the electrons with the commutative diagrams similar to Fig. 11 and the other associated with the electrons with the diagrams similar to Fig. 12. Each of the final position subsets exhibits a statistical distribution of a single-slit diffraction.

\section{DISCUSSION}

As shown in this paper that $t$-topos can be very useful in describing mathematical structures for the foundation of physical particles in quan- 
tum mechanics. In the language of $t$-topos, an electron is represented by a presheaf $e$. Contradictory particle-like properties and wave-like properties of electron are consistent with the model based on a presheaf together with a choice of an object in the $t$-site. When the electron is actually observed, an object $V$ in the site $\mathcal{S}$ is selected, and the electron is localized as $\underline{e}=e(V)$. If an object is not specified, in other words, no observation is made, the electron remains in the wave ur-state $e(-)$.

In the electron double-slit experiment, the electron remains in the wave ur-state if both slits are open and no observation is made at the slit because there are more than one choice for the object in the $t$-site. This type of experiment is represented by the fundamental commutative diagram in Fig. 10. The particle ur-state of the electron on the screen $e(U)$ is dependent on the factorization of the morphism from $V$ to $U$. For the double-slit experiment, there are two objects $W_{A}$ and $W_{B}$ associated the slits, and both of them affect $U$. Closing one of the slits or detecting the electron at the slit selects a particular object, and thus the electron is in a localized particle ur-state at the slit. The fundamental commutative diagram reduces to simpler diagrams (Fig. 11 or Fig. 12). The double-slit interference pattern disappears in this case because $e(U)$ is dependent on only one intermediate object (either $W_{A}$ or $W_{B}$, but not both).

In this paper, we did not presheafify physically observable quantities other than time and space, but we could have introduce presheaves associated with energy and momentum of an electron at the microcosm (quantum) level $\mathcal{C}_{1}$. Some presheaves may be coupled together. For example, the space and momentum presheaves form a pair $(\kappa, \pi)$ acting as one object of the $t$-topos. This may allow us to interpret the uncertainty principle from the $t$-topos point of view. Spin too can be presheafified. When an object $V$ in the $t$-site is specified, $\sigma(V)$ gives us a discrete number for the spin of the electron. If we do not specify the object, then we have $\sigma(-)$.

Also missing is dynamics of electron in terms of $t$-topos as it travels from the initial position with associate object $V$ to the final position with object $U$. These details will be addressed in our second paper on $t$-topos and the electron double-slit interference. It will be shown that how the interference patter on the screen changes, as wavelength, slit width or slit spacing increases or decreases, is consistent with properties of presheaf.

\section{REFERENCES}

1. R. P. Feynman, R. B. Leighton, and M. Sands, The Feynman Lectures on Physics, Vol. III (Addison-Wesley, Reading, MA, 1965). 
2. S. I. Gelfand and Y. I. Manin, Methods of Homological Algebra (Springer, Berlin, 1996).

3. G. Kato, The Heart of Cohomology (Springer, Berlin, 2006).

4. G. Kato, Kohomorojii No Kokoro (Iwanami Shoten, Tokyo, 2003).

5. G. Kato, "Presheafification of matter, space and time (the notion of $t$-topos)," in Invited talk at The Second International Workshop on Sheaves and Topos for Theoretical Physics at Imperial College, London, July 17-18, 2003.

6. G. Kato, "Elemental principles of temporal topos," Europhys. Lett. 68(4), 467-472 (2004).

7. G. Kato, "Elemental t.g. principles of relativistic $t$-topos (presheafification of matter, space, and time)," Europhys. Lett. 71(2), 172-178 (2005).

8. S. Roy, "Planck scale physics, pregeometry and the notion of time," in The Nature of Time: Geometry, Physics and Perception, the Proce. of the NATO Advanced Research Workshop, Tantanská Lomnica, May 21-24, 2002, R. Buccheri, M. Saniga, and W. M. Stuckey, eds. (NATO Science Series II 95, Kluwer, Dordrecht, 2003).

9. G. Kato, M. Kafatos, S. Roy, and T. Tanaka, "Sheaf cohomology and geometrical approach to EPR non-locality," in preparation.

10. C. J. Isham and J. Butterfield, "A topos perspective on the Kochen-Specker theorem: I. Quantum states as generalized valuations," Int. J. Theor. Phys. 37(11), 2669-2733 (1998).

11. J. Butterfield and C. J. Isham, "A topos perspective on the Kochen-Specker theorem: II. Conceptual aspects and classical analogues," Int. J. Theor. Phys. 38(3), 827-859 (1999).

12. J. Hamilton, C. J. Isham, and J. Butterfield, "A topos perspective on the Kochen-Specker theorem: III. Von Neumann algebras as the base category," Int. J. Theor. Phys. 39(6), 14131436 (2000).

13. J. Butterfield and C. J. Isham, "A topos perspective on the Kochen-Specker theorem: IV. Interval valuations," Int. J. Theor. Phys. 41(4), 613-639 (2002). 